\title{
The human ZBP-89 homolog, located at Chromosome 3q21, represses gastrin gene expression
}

\author{
D.J. Law, ${ }^{1}$ S.A. Tarlé, ${ }^{1}$ Juanita L. Merchant ${ }^{1,2}$ \\ ${ }^{1}$ Howard Hughes Medical Institute, University of Michigan, Ann Arbor, Michigan 48109, USA \\ ${ }^{2}$ Departments of Internal Medicine and Physiology, 1150 West Medical Drive, MSRBI, 3510, University of Michigan, \\ Ann Arbor, Michigan 48109-0650, USA
}

Received: 8 July 1997 / Accepted: 22 October 1997

Rat $Z B P-89$ cDNA encodes an $89-\mathrm{kDa}$, zinc finger protein that binds a GC-rich element in the human gastrin promoter. This element modulates both basal- and epidermal growth factor (EGF)-induction of gastrin gene expression (Merchant et al. 1995). Coexpression of ZBP-89 and gastrin reporter constructs blocks EGF induction and represses basal gastrin gene expression (Merchant et al. 1996). Rat ZBP-89 similarly binds to and represses the activity of the ornithine decarboxylase (ODC) promoter, concomitantly inhibiting cell proliferation (Remington et al. 1997). ZBP-89 shares $99 \%$ amino acid sequence identity with two closely related mouse homologs, G-rich box-binding protein (GRBBP; Passantino et al. 1996) and BFCOL1 (Hasegawa and de Crombrugghe 1997). Mutations of the BFCOL1 binding site in the pro $\alpha 2$ (I) collagen promoter inhibit BFCOL1 binding and result in increased promoter activity (Hasegawa and de Crombrugghe, 1997). Collectively, these data show that rodent $Z B P$-89-related genes act as transcriptional repressors by binding to specific GC-rich promoter elements, and, at least in some cases, this activity exerts a regulatory effect on cell proliferation.

The human cDNA, ht $\beta$, shares about 90\% DNA sequence identity with the open reading frames of rodent $Z B P-89$ homologs. However, two single-nucleotide deletions in ht $\beta$ cDNA, $3^{\prime}$ of the zinc finger domain, cause premature stop codons. As a result, ht $\beta$ encodes a 494 rather than a 794-amino acid protein (Merchant et al. 1996; Wang et al. 1993). The truncated, 49-kDa, ht $\beta$ protein acts as a transcriptional activator, moderately activating T-cell receptor promoters (Wang et al. 1993). The structural and functional comparisons of rodent $Z B P-89$ homologs with ht $\beta$ suggested to us that the point deletions that distinguish ht $\beta$ may have originated as somatic mutations of wild type human ZBP-89 in the Jurkat cells from which the ht $\beta$ cDNA was isolated. To test this hypothesis, we isolated a full-length $(\sim 89 \mathrm{kDa})$ human ZBP-89 cDNA from a normal leukocyte library, compared genomic localization of the corresponding gene with that of ht $\beta$, and determined its effects on gastrin gene expression. We report that full-length ZBP-89 maps to Chr 3q21 where the ht $\beta$ cDNA was mapped (Schuler et al. 1996). Furthermore, like its rat homolog, human ZBP-89 functions as a repressor of gastrin gene expression.

The human ZBP-89 cDNA clone hZBP-89-16 contains a 2.4$\mathrm{kb}$ insert and was isolated from a SuperScript (Life Technologies) leukocyte library by positive selection (GeneTrapper, Life Technologies) with oligonucleotides from ht $\beta$. The open reading frame of hZBP-89-16 encodes a 794-amino acid protein, similar to the rat and mouse homologs, and lacks the internal stop codons of ht $\beta$. A 600-bp, EcoRV fragment from the 5'-UTR of ZBP-89-16 cDNA was used to screen a human BAC library (Genome Systems, St. Louis, Mo.), and overlapping clones B469N19, B460C18, and B502J22 were isolated (Fig. 1). A subcloned 1.2-kb EcoRI frag-

Correspondence to: J.L. Merchant ment from B469N19 was sequenced and used to develop an STS (374F/794R) for PCR-based screening of the human CEPH YAC DNA matrix pools (Research Genetics, Huntsville, Ala.). The resulting YAC/BAC contig shows that the human ZBP-89 gene maps to chromosome band 3q21 (144-146 cM on the genetic map) and, more precisely, is within $100 \mathrm{~kb}$ of D3S1551. This agrees with our FISH data showing hybridization to $3 \mathrm{q} 21$ using BAC B469N19 as a probe (data not shown). This localization is also the same as reported for ht $\beta$ (Schuler et al. 1996), suggesting that two alternative forms of human ZBP-89-related protein are derived from a single locus. To better assess this possibility, we analyzed the coding sequences contained in the ZBP-89 BAC clones.

The ZBP-89 DNA sequence derived from BAC B469N19 was compared with those of related genes (Fig. 2). The human sequence shares $97 \%$ amino acid sequence identity with correspond-

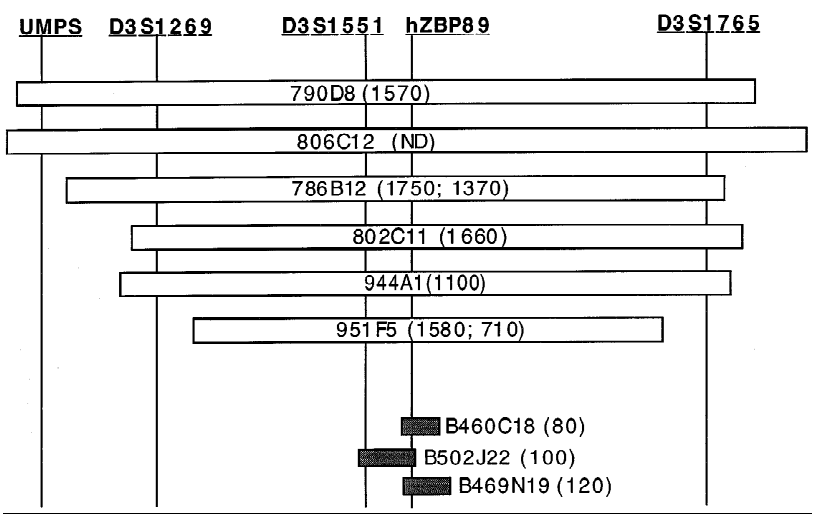

Fig. 1. Genomic clone contig encompassing human ZBP-89 is linked to markers at $\mathrm{Chr} 3 \mathrm{q} 21$. Hybridization probes for screening genomic libraries were derived from human cDNA clone hZBP-89-16 (GenBank Accession number AF039019). This clone contains a 2.4-kb insert and was isolated from a SuperScript (Life Technologies) leukocyte library by use of the Gene-Trapper method (Life Technologies) and primers HTB-1 (TCAAGATCGAAGTATGCCTCAC), HTB-2 (GCTCTGAGGAAGATTCTGGGC), and HTB-3A (TGCCTTCTGAGTCCAGTAAAG). The CEPH YAC library was screened by PCR analysis of matrix DNA pools obtained from Research Genetics, with intra-exonic primers BAC374F (CACCCACCTAAGTTAGTTCTCAAAA) and BAC794R (ACATTCAGTGCCTGTGACTCTAATA), which comprise STS hZBP-89. Database analysis showed that clone $806 \mathrm{C} 12$ is contained within Whitehead Institute YAC contig WC-947 (www-genome.wi.mit.edu). Additional STS content analysis resulted in the revised YAC and BAC genomic clone contig shown here. Size estimates $(\mathrm{kb})$ are indicated for YAC (open bars) and BAC (solid bars) clones. Map is not to scale, since extent of chimerism in YAC clones is not known. Accession numbers for the markers are: UMPS (GDB: 181231), D3S1269 (GDB:188016), D3S1551 (GDB:199164), D3S1765 (GDB:686679), and hZBP-89 (GenBank U96633). The centromere is to the left. 
HSAZBP :

HSAHTB :

RNOZBP :

GRBBP :

BFCOL1 :

HSAZBP :

HSAHTB :

RNOZBP :

GRBBP :

BFCOL1:

HSAZBP :

HSAHTB :

RNOZBP :

GRBBP :

BFCOL1:

HSAZBP :

HSAHTB :

RNOZBP :

GRBBP :

BFCOL1:

HSAZBP :

RNOZBP :

GRBBP :

BFCOL1:

HSAZBP :

RNOZBP :

GRBBP :

BFCOL1:

HSAZBP :

RNOZBP :

GRBBP :

BFCOL1:
QYFSRTDRVLKHKRMCHENHDKKLNRCAIKGGLLTSEEDSGFSTSPKDNSLPKKKRQKTE

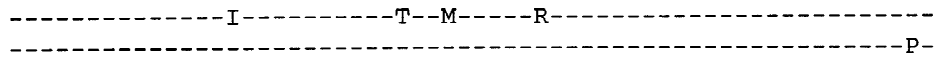

$-\mathrm{N}-$

$-\mathrm{P}----\mathrm{Q}--$

KKSSGMDKESALDKSDLKKDKNDYLPLYSSSTKVKDEYMVAEYAVEMPHSSVGGSHLKDA

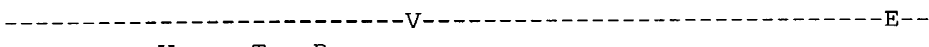

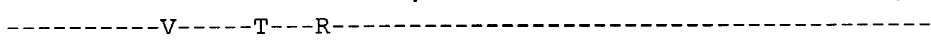

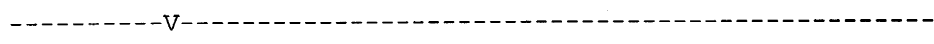

$\Delta$

SGEIHPPKLVLKKINSKRSLKQPLEQNQTISPLSTYEESKVSKYAFELVDKQALLDSEGN

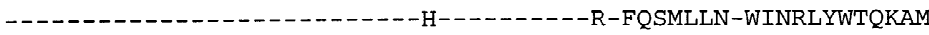

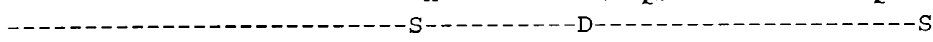

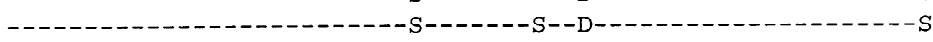

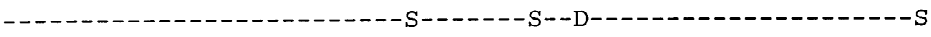

$\Delta$

ADIDQVDNLQEGPSKPVHSSTNYDDAMQFLKKKRYLQAASNNSREYALNVGTIASQPSVT LTLIRLIICRRAQ*

-

QAAVASVIDESTTASILESQALNVEIKSNHDKNVIPDEVLQTLLDHYSHKANGQHEISFS

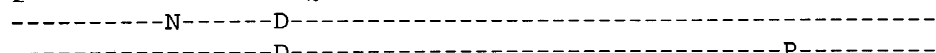

- - - -

VADTEVTSSISINSSEVPEVTPSENVGSSSQASSSDKANMLQEYSKFLQQALDRNSQNDA
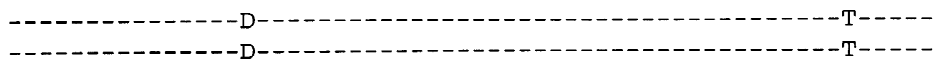

YLNSPSLNFVTDNQTLPNQPAFSSIDKQVYATMPINSFRSGMNS

-

-

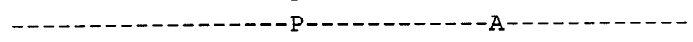

Fig. 2. Comparison of partial peptide sequences among mammalian $Z B P$-89-related genes. Amino acid identity is indicated by a dash (-), and the positions of the single nucleotide deletions in the ht $\beta$ sequence are indicated by $\Delta$. The sources for the sequences are: human ZBP-89 (HSAZBP-89), this report, GenBank U96633; human ht $\beta$ (HUMTB), GenBank L04282; rat ZBP-89 (RNOZBP), GenBank U30381; mouse G-rich box binding protein (GRBBP), EMBL X98096; mouse transcription factor BFCOL1, GenBank U80078. The homologies were identified by BLAST analysis (Altschul et al. 1990). The human sequence was derived from a 1.2-kb EcoRI subclone of ZBP-89 BAC clone B469N19, with standard automated cycle sequencing. ing segments of rat ZBP-89 cDNA (Merchant et al. 1996) and the two related mouse cDNAs. GRBBP (Passantino et al. 1996) and BFCOL1 (Hasegawa and de Crombrugghe, 1997), confirming that the BAC clones encompass the human ZBP-89 homolog. This 404-amino acid segment spans one zinc finger and the C-terminal domain of ZBP-89, indicating that these functional domains are included in a single exon. The sequence comparisons also showed striking homology ( $\geqslant 92 \%$ DNA sequence identity) between human ZBP-89 and a number of expressed sequence tags (ESTs). Homologous ESTs were derived from cDNAs prepared from the following tissues: human 12-week embryo (Accession number AA329458), human 20-week fetal liver and spleen (T81259), human insulinoma (U09581), human olfactory epithelium (H70710), human melanocyte (N26148), human tonsillar cells (AA286728), human adult skeletal muscle (AA192128), mouse 12 d.p.c. fetal limb (X98096), mouse 14 d.p.c. embryo fibroblasts (U80078), mouse 4-week thymus (AA125037), newborn mouse kidney (AA241357), and rat pancreatic islets (C06563).

The reading frameshifts that result in multiple upstream stop codons in ht $\beta$ (Merchant et al. 1996; Wang et al. 1993) were not present in the BAC clone sequence. We also failed to amplify the ht $\beta$ stop codons from human genomic DNA using primers spanning the region (data not shown). This suggests that the single base-pair deletions and resulting stop codons in ht $\beta$ cDNA are unique to that clone and possibly arose by somatic mutation. Southern blot analysis of genomic DNA showed that ZBP-89 cDNA probes detect a single locus (data not shown). Since we previously reported that full-length rat ZBP-89 functioned as a repressor of gastrin gene expression (Merchant et al. 1996), we next analyzed the effect of the human ZBP-89 homolog on gastrin expression.
Figure 3 shows the effect of human ZBP-89 co-expression on human gastrin reporter constructs transfected into Drosophila Schneider SL2 cells. Human ZBP-89 cDNA expresses full-length ZBP-89 protein from the CMV promoter in pCMV-SPORT3 (Life Technologies). Human ZBP-89 expression reduced basal gastrin promoter activity by $50 \%$ when compared with a vector control. The repression was slightly greater than that of rat ZBP-89, which resulted in $40 \%$ reduction in basal promoter activity. Thus, the full-length human ZBP-89 protein, like its rat homolog, represses gastrin gene expression.

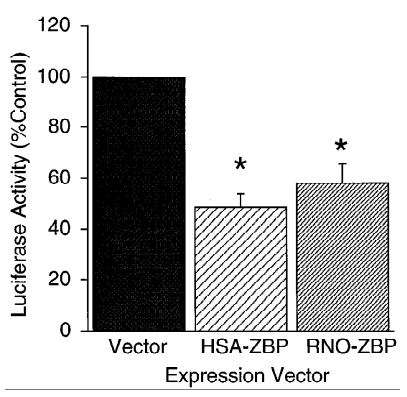

Fig. 3. Human ZBP-89 inhibits gastrin gene expression in cultured Schneider cells. Drosophila embryo cell line SL2 (ATCC CRL-1963; Schneider, 1972) was maintained in Schneider's Drosophila medium (GIBCO \#11720) supplemented with $10 \%$ heat-inactivated fetal calf serum, and incubated at $22^{\circ}-24^{\circ} \mathrm{C}$. Transient transfections were performed by the calcium phosphate coprecipitation method. Each trans fection reaction contained $2 \mu \mathrm{g}$ of expression construct and $0.16 \mu \mathrm{g}$ of 240 GasLuc reporter in pGL2-Basic (Merchant et al. 1996). Expression constructs were: HSA-ZBP-2.4 kb human ZBP-89 cDNA in pCMV-SPORT3; RNO-ZBP-2.4 kb rat ZBP-89 cDNA in pBKCMV (Merchant et al. 1996); and Vector-insertless pBKCMV. Combined $\beta$-galactosidase and luciferase reporter gene assays were performed by the Tropix (Bedford, Mass.) Dual-Light assay. The data represent the averages of three independent experiments. *indicates statistically significant at $\mathrm{P}<0.001$ (student's $\mathrm{T}$ test). 
Collectively, the data presented here show that a locus at human Chr 3q21 encodes an 89-kDa ZBP-89 homolog, which shares $97 \%$ amino acid sequence identity with the rat and mouse genes. Like rat ZBP-89, the human protein acts as a repressor of gastrin gene expression. Although ht $\beta$ (Schuler et al. 1996) and human ZBP-89 (reported here) both map to Chr 3q21, no evidence of the ht $\beta$ frameshift mutations could be found either in the genomic and cDNA clones we isolated or in sequences amplified from total genomic DNA. It is possible that the ht $\beta$ frameshifts resulted from somatic mutations of ZBP-89 in the Jurkat, acute T-cell leukemia cell line used to isolate ht $\beta$ cDNA. Similar types of point deletions in the GLI3 gene, downstream of the zinc finger region, result in truncated GLI3 protein associated with Pallister-Hall syndrome (Kang et al. 1997). This hypothesis further suggests that the altered function of ht $\beta$ protein (transcriptional activator versus repressor) may be involved in the molecular etiology of T-cell leukemia. Truncated proteins resulting from point deletions have been identified in several human malignancies, including leukemias (Fenaux et al. 1991; Kurosawa 1994; Kawamura et al. 1995) and solid tumors (Kishimoto et al. 1992; Hogervorst et al. 1995; Slavc et al. 1995). Interestingly, the ZBP-89 locus maps just proximal to a leukemia break point cluster region at Chr 3q21 (Pekarsky et al. 1995). The possibility that mutations in human ZBP-89 may be involved in oncogenesis is further supported by the fact that rat $Z B P-89$ regulates cell proliferation (Remington et al. 1997), and that ZBP-89 expression is altered in human solid tumors (Taniuchi et al. 1997). Further analysis of human ZBP-89 structure and function will clarify its role in regulation of cell proliferation.

Acknowledgments. This work was supported by U.S. Public Health Service grant DK-45729 (J.L. Merchant). Dr. Merchant is an investigator of the Howard Hughes Medical Institute. Pia Sullivan provided technical support, and Gail Kelsey provided administrative support.

\section{References}

Altschul SF, Gish W, Miller W, Myers EW, Lipman DJ (1990) Basic local alignment search tool. J Mol Biol 215, 403-410

Fenaux P, Jonveaux P, Quiquandon I, Lai JL, Pignon JM, LoucheuxLefebvre MH, Bauters F, Berger R, Kerckaert JP (1991) P53 gene mutations in acute myeloid leukemia with 17 p monosomy. Blood 78, 1652 1657

Hasegawa T and de Crombrugghe B (1997) Cloning and characterization of a transcription factor that binds to the proximal promoters of the two mouse type I collagen genes. J Biol Chem 272, 4915-4923

Hogervorst FB, Cornelis RS, Bout M, van Vliet M, Oosterwijk JC, Olmer
R, Bakker B, Klijn JG, Vasen HF, Meijers-Heijboer H, et al. (1995) Rapid detection of BRCA1 mutations by the protein truncation test. Nat Genet 10, 208-212

Kang S, Graham JM Jr., Olney AH, Biesecker LG (1997) GLI3 frameshift mutations cause autosomal dominant Pallister-Hall syndrome. Nat Genet $15,266-268$

Kawamura M, Kikuchi A, Kobayashi S, Hanada R, Yamamoto K, Horibe K, Shikano T, Ueda K, Hayashi K, Sekiya T, et al. (1995) Mutations of the p53 and ras genes in childhood $t(1 ; 19)$-acute lymphoblastic leukemia. Blood 85, 2546-2552

Kishimoto Y, Murakami Y, Shiraishi M, Hayashi K, Sekiya T (1992) Aberrations of the p53 tumor suppressor gene in human non-small cell carcinomas of the lung. Cancer Res 52, 4799-4804

Kurosawa M (1994) Molecular study on minute alterations of the p53 and the N-ras genes in hematologic malignancies. Hokkaido J Med Sci 69, 543-554

Merchant JL, Shiotani A, Mortensen ER, Shumaker D, Abraczinskas D (1995) Epidermal growth factor stimulation of the human gastrin promoter requires Sp1. J Biol Chem 270, 6314-6319

Merchant JL, Iyer GR, Taylor BR, Kitchen JR, Mortensen ER, Wang Z, Flintoft RJ, Michel JR, Bassel-Duby R (1996) ZBP-89, a Krüppel-like zinc finger protein, inhibits epidermal growth factor induction of the gastrin promoter. Mol Cell Biol 16, 6644-6653

Passantino R, Antona V, Barbieri G, Feo S, Giallongo A (1996) M. musculus mRNA for G-rich box-binding protein. GenBank Entry Accession number X98096.

Pekarsky Y, Zabarovsky E, Kashuba V, Drabkin H, Sandberg AA, Morgan R, Rynditch A, Gardiner K (1995) Cloning of breakpoints in 3q21 associated with hematologic malignancy. Cancer Genet Cytogenet 80, 1-8

Remington MC, Tarlé SA, Simon B, Merchant JL (1997) ZBP-89, a Krüppel-type zinc finger protein, inhibits cell proliferation. Biochem Biophys Res Commun 237, 230-234

Schneider I (1972) Cell lines derived from late embryonic stages of Drosophila melanogaster. Embryol Exp Morphol 27, 353-365

Schuler GD, Boguski MS, Stewart EA, Stein LD, Gyapay G, Rice K, White RE, Rodriguez-Tome P, Aggarwal A, Bajorek E, Bentolila S, Birren BB, Butler A, Castle AB, Chiannilkulchai N, Chu A, Clee C, Cowles S, Day PJ, Dibling T, Drouot N, Dunham I, Duprat S, East C, Hudson TJ, et al. (1996) A gene map of the human genome. Science 25, 540-546

Slavc I, MacCollin MM, Dunn M, Jones S, Sutton L, Gusella JF, Biegel JA (1995) Exon scanning for mutations of the NF2 gene in pediatric ependymomas, rhabdoid tumors and meningiomas. Int J Cancer 64, 243-247

Taniuchi T, Mortensen ER, Ferguson A, Greenson J, Merchant JL (1997) Overexpression of $Z B P$ - 89 , a zinc finger DNA binding protein, in gastric cancer. Biochem Biophys Res Commun 233, 154-160

Wang Y, Kobori JA, Hood L (1993) The ht $\beta$ gene encodes a novel CACCC box-binding protein that regulates T-cell receptor gene expression. Mol Cell Biol 13, 5691-5701 